\title{
Renate Augstein, Ministerialdirektorin
}

\author{
Juristinnen machen Karriere - wir stellen sie vor
}

Das Gespräch führte Prof. Dr. Dagmar Oberlies Anfang Januar 2013.

Kannst du dich noch daran erinnern, wann und warum du in den djb eingetreten bist?

Ja. Ich war, ich schätze so 1980/81, für die Friedrich-Naumann-Stiftung auf einer rechtspolitischen Tagung in Hannover. Ich weiß aber nicht mehr, um was es dabei genau ging. Lore Maria Peschel-Gutzeit hatte den djb dort vertreten. Sie hat hervorragend argumentiert und mir so gut gefallen, dass ich dachte, diesen djb muss ich mir merken, das scheint ein interessanter Verein zu sein! Danach bin ich dann auch tatsächlich Mitglied geworden. Das lag eindeutig an der Person Peschel-Gutzeit!

In Vorbereitung auf dieses Gespräch hat mich der Unterschied in unseren Zugängen interessiert ...

... den größten Unterschied hast du mal selbst benannt: Du bist eine Sprinterin und ich bin eine Langstreckenläuferin ...

... stimmt, sag ich immer, dass ich die Frau der ersten 100 Meter bin ...

Ja, und ich bin die Ausdauernde.

Dazu gehört meine Beobachtung, dass wir ja bei gleichen Themen gelandet sind, aber du, so kommt es mir vor, früher auf dem Marsch durch die Institutionen ...

Ich überlege, wie ich an die Frauenpolitik gekommen bin ... Interessiert war ich eigentlich lange. Schon auf der Mädchenschule, auf der ich war, hatten wir frauenpolitische Diskussionen. Aber es stimmt, ich kam nicht als „Bewegungsfrau“.

Ich glaube, die Initialzündung kam über eine sehr feministisch orientierte Kollegin bei der Friedrich-Naumann-Stiftung, die mich dort, in meiner ersten Stelle als Volljuristin, eingearbeitet und zu frauenpolitischen Veranstaltungen mitgenommen hat. Ich erinnere noch gut die Treffen der Initiative 6. Oktober, die sich - nach der Bundestagswahl am 5.Oktober 1980 - in Bonn gegründet hat, weil so wenig frauenpolitische Forderungen umgesetzt wurden. Dadurch hatte ich früh Kontakt zu Frauen wie Barbelies Wiegmann, Herrad Schenk, Birgit Meyer u.a., die in Bonn feministisch unterwegs waren. Also meine frauenpolitische Sozialisierung kam stark über diese Bonner Frauenszene, in der ich gut verankert war.

Damals hatte ich auch, auf Einladung von Birgit Meyer, die heute Professorin in Esslingen ist, am Frauenhandlexikon mitgeschrieben, was eine wichtige Erfahrung war und, nach wie vor, für mich eine wichtige Quelle ist. Weitere frauenpolitische Buchbeiträge folgten dann.

Gerade für die jüngeren Kolleginnen könnte ja interessant sein, was man deines Erachtens mitbringen muss, um in und über
Geboren 1950 in Köln absolvierte Renate Augstein nach dem Abitur an der Kölner Ursulinenschule die Rechtspflegeausbildung am Oberlandesgericht Köln, anschließend studierte sie Jura an der Universität zu Köln. Nach dem zweiten juristischen Staatsexamen 1979 arbeitete sie zunächst als gesellschaftspolitische Referentin bei der FriedrichNaumann-Stiftung in Bonn, bevor sie Ende 1981 zum damaligen Bundesministerium für Familie, Jugend und Gesundheit wechselte. Dort arbeitete sie in verschiedenen Funktionen, seit 2012 als Leiterin der Abteilung Gleichstellung, Chancengleichheit. Arbeitsschwerpunkte waren insbesondere die Bekämpfung von Gewalt gegen Frauen, ferner die Implementierung von Gender Mainstreaming in die Arbeit der Bundesregierung. Als Sonderaufgaben waren ihr die Umsetzung der EU-Gleichbehandlungsrichtlinien mit dem Aufbau der Antidiskriminierungsstelle des Bundes sowie die Entwicklung des Programms „Perspektive Wiedereinstieg“ übertragen.

Renate Augstein ist Autorin verschiedener juristischer, rechtsund gleichstellungspolitischer Veröffentlichungen.

Sie ist ehrenamtlich engagiert u.a. bei der Evangelischen Kirche in Deutschland, beim Deutschen Juristinnenbund, beim Institut für Opferschutz im Strafverfahren e.V. „Recht Würde Helfen“, Berlin, und beim „Moabiter Theaterspektakel e.V.“, Berlin.

Renate Augstein bekleidet folgende ehrenamtliche Positionen:

- Mitglied des Präsidiums der Evangelischen Aktionsgemeinschaft für Familienfragen,

- Mitglied des Aufsichtsrats des Evangelischen Zentralinstituts für Familienberatung.

Institutionen - Parteien, Stiftungen, Behörden, Verbände Frauenpolitik zu machen?

Ich glaube nicht, dass man das verallgemeinern kann. In der (FDP-nahen) Friedrich-Naumann-Stiftung, damals eine eher sozialliberale Organisation, habe ich z.B. Seminare zu Frauenthemen abgehalten: Frauen und Wohnen, Frauen und Medien, Frauenhäuser, Geschichte der Frauenbewegung, Antidiskriminierungsgesetz, $\mathbb{} 218$ StGB ... Das alles gehörte damals zu meinen Themen.

Ich würde mich als Person definieren, die versucht, eine Verbindung herzustellen zwischen der Basis-Bewegung draußen und der Politik in Verbänden, Parteien und Parlament. Ich übersetze die Forderungen und trage sie in die Politik hinein. An dieser Schnittstelle habe ich mich gut aufgehoben gefühlt. Ich spreche sozusagen beide Sprachen.

Denkst du, dass man auch pragmatisch sein muss - jedenfalls pragmatischer als die autonome Frauenbewegung der damaligen Zeit? 
Auf jeden Fall kann ich pragmatisch sein; bin ich auch immer mehr geworden.

Aber ich glaube, damals war ich es nicht, da war ich sehr viel radikaler und glaubte, die Welt verändern zu können. Mit dem Nichtdurchsetzen von Wahlversprechen 1980 und dem Niedergang der sozialliberalen Koalition kam für mich eine große Desillusionierung. Das war ein tiefer Einschnitt, fast eine Art Depression. Wir hatten ja diese ganzen Themen in das FDP-Wahlprogramm 1980 reinbekommen: Fristenlösung, Antidiskriminierungsgesetz, Streichung von $\mathbb{} 175$ StGB, Gleichstellung nichtehelicher Lebensgemeinschaften etc., da bin ich heute noch stolz darauf! Damals wurde leider nichts davon umgesetzt, das hatte mir sehr zugesetzt. Doch viele der Themen haben mich weiterbegleitet und in den Folgejahrzehnten konnte doch noch Einiges verwirklicht werden - das hat sicher zu meinem heutigen Pragmatismus beigetragen und ich wurde zur „Langstreckenläuferin“.

\section{Was muss man wissen, um in Institutionen Politik zu machen?} Man muss wissen, wie Institutionen ticken. Und man muss das institutionelle Handwerkszeug im Sinne der Sache einsetzen können.

Außerdem: Institutionen sind träge, das kann man sich zunutze machen. Wenn ich die Sachen in die Hand nahm und loslegte, dann kam ich oft ziemlich weit. Es machte eben sonst keiner und oft war man froh, dass es „einen Dummen gab“, die Erfolge nahm man gerne mit. Also, wer Arbeit sucht und macht, ist in Institutionen nicht schlecht aufgehoben.

\section{Wie wichtig war und ist es, auch Parteierfahrung zu haben?}

Schon während meiner Tätigkeit bei der Friedrich-NaumannStiftung hatte ich viel Kontakt mit der Fraktion, mit Fachausschüssen und Arbeitskreisen. Da habe ich gelernt, wie Politik funktioniert und das war wichtig. Obwohl ich in der damaligen desillusionierten Phase auch dachte, ich muss raus aus der Politik. Ich wollte weg von der Stiftung und überlegte, Arbeitsrichterin zu werden. Aber dann habe ich gemerkt, so ganz lassen kann ich es nicht: Ich wechselte deshalb ins Familienministerium, wo ich zunächst mal nichts mehr mit „der großen Politik“ zu tun hatte, sondern als Referentin juristisch im Bereich Apothekenrecht und Gifte arbeitete.

Zum Gewaltthema kommen wir gleich noch. Zuerst interessiert mich noch eine Stelle in deinem Lebenslauf. Ich habe mal nachgerechnet: Du müsstest so um 1968 Abitur gemacht haben ...

1969.

Danach hast du eine Ausbildung als Rechtspflegerin gemacht, heißt Grundbuchamt, Nachlassgericht ... Warum tut jemand so was?

Nicht unbedingt, weil es meinen Wünschen entsprach. Ich wollte damals entweder Physik studieren oder Journalistin werden. Ein Eignungstest beim Arbeitsamt hatte aber ergeben, dass ich von Physik bitte die Finger lassen sollte, weil meine mathematischen Fähigkeiten doch sehr zu wünschen ließen, auch wenn ich in Physik in der Schule sehr gut war. Meine Eignungskurve empfahl mich stattdessen für ein Jurastudium. Daran hatte ich vorher keine Sekunde gedacht! Aber als ich hörte, dass ich damit auch Journalistin - und vieles mehr werden kann, habe ich mich dafür entschieden. Allerdings bestand mein Vater darauf, zuerst eine Ausbildung abzuschließen („weil ja die meisten Frauen nicht zu Ende studieren und heiraten“); danach könnte ich dann immer noch studieren.

\section{... Jura light, gefolgt von Jura heavy}

Nein, ich würde sagen, die Rechtspflegerei war „Jura heavy“: Unser Rechtspflegeexamen hatte das gleiche Niveau wie das 1. Staatsexamen. Im Jurastudium haben mich meine RundumKenntnisse aus der Rechtspflegeausbildung getragen. Durch sie hatte ich die Zusammenhänge begriffen; im Jurastudium hätte ich sonst so schnell keinen Durchblick gehabt.

Ich frag dich mal nicht, ob das dein Karrieretipp für die Juristin von heute ist ...

... wäre es, durchaus. In dieser sehr umfassenden Ausbildung wurden Zusammenhänge hergestellt, das war im Jurastudium nicht so. Und auch der Praxisbezug hat mir geholfen, vieles besser zu verstehen.

Du hast sehr lange - und sehr erfolgreich - das Thema „Gewalt gegen Frauen" bearbeitet. Was sind aus deiner Sicht „Milestones“, die in deiner Zeit der Befassung mit dem Thema erreicht wurden? Wo du auch denkst, du warst zur richtigen Zeit am richtigen Ort oder auch, dass deine Ausdauer, von der ja schon die Rede war, etwas bewirkt hat?

Ja, gibt es.

Dazu gehört der Aufbau der Hilfe-Infrastruktur in den Ländern, vorbereitet und vorangetrieben durch die vom Bund geförderten Modellprojekte, also die Frauenhäuser, die Notrufe und Interventionsstellen bis hin zu speziellen Beratungsstellen. In der „Nachwendezeit“ 1982 ff. gab es starke Bestrebungen, die verbandlichen Kinder- und Frauenschutzhäuser stärker zu fördern, zu Lasten der autonomen Frauenhäuser. Damals habe ich, nach langem Nachdenken, eine Leitungsvorlage gemacht, in der ich argumentiert habe, dass der Bund für ein plurales Angebot sorgen muss und nicht einzelne Einrichtungen bevorzugen darf. Dem hat der damalige Minister Heiner Geißler dann auch zugestimmt, was allerdings zu der kuriosen Situation führte, dass ich in der Folge genau darauf achten musste, immer abwechselnd ein Projekt an die Verbandshäuser und das nächste an die autonome Richtung zu vergeben. Das war ein Balanceakt (und der Sache vielleicht nicht immer wirklich dienlich), bei dem ich aber in beiden Bereichen ein gutes Standing hatte. Das wiederum war sehr hilfreich für die Anti-Gewalt-Politik.

Das andere ist der lange Atem bei Gesetzgebungsvorhaben. Wobei ich sagen muss, dass die letztendlichen Erfolge nicht nur meiner zähen Arbeit zu verdanken waren. Hätte damals, bei der Diskussion um die Strafbarkeit in der Ehe, nicht 
Irmgard Karwatzki (MdB, CDU) die Frauen der Fraktionen zu einem Gruppenantrag des Parlaments zusammengebracht, dann wäre das wahrscheinlich heute noch nicht durch. Übrigens war auch die Strafrechtskommission des djb sehr aktiv und hatte einen Diskussionsentwurf zu $\mathbb{\$} 177$ StGB erarbeitet, der auch Pate gestanden hatte ... Oder die Abschaffung des \175 StGB: Etwas, das zum Beispiel unter einem Justizminister Hans-Jochen Vogel überhaupt nicht möglich war, ist nach der Wiedervereinigung fast unbemerkt gelungen.

... ich glaube, eingeleitet durch eine Bundesratsinitiative aus Hamburg, wo Peschel-Gutzeit Justizministerin war ...

Das kann gut sein. Was ich sagen will, manchmal ist einfach die Zeit reif oder es kommen andere Konstellationen und plötzlich klappt etwas, woran man vorher jahrzehntelang gearbeitet hat. Und trotzdem denke ich, auch diese ganze Vorarbeit war wichtig.

Wenn man sich so lange mit dem Thema Gewalt befasst, fragen Menschen schon mal, wie man das aushält. Wir, die die Arbeit machen, wissen, dass sie einem auch etwas geben kann. Was hat sie dir gegeben?

Dass ich nicht ausbrannte, hat sicher auch damit zu tun, dass ich keine persönlichen Beratungen gemacht habe. Ich war stümmelung oder Zwangsverheiratung. Ein Riesenthemenbereich und wenn es in einem Bereich mal nicht lief, ging es sicher irgendwo anders weiter. Außerdem hat man es mit einer Bandbreite an Instrumenten zu tun, von Projektförderung über Öffentlichkeitsarbeit und Kampagnen hin zu gesetzlichen Regelungen und begleitender Forschung. Und schließlich kooperiert man in diesem Feld mit sehr verschiedenen Akteuren und Akteurinnen. Was mich z.B. beflügelt, sind „meine“ BundLänder-Arbeitsgruppen. Die erste hatte ich schon 1987 zum Thema Frauenhandel ins Leben gerufen. Dort sitzen NGOs neben Bundesressorts, Ländern und dem BKA, da fließt so viel Kompetenz zusammen. Es war mein Ehrgeiz, daraus was zu machen, auch wenn es manchmal mühselig war oder aussah als würde es gar nicht weitergehen, aber dann kam doch wieder ein neuer Impuls.

Ein „Erfolg“, der sich feministisch nicht so eindeutig bejubeln lässt, war die Einführung des Prostitutionsgesetzes, an der du ja maßgeblich beteiligt warst. Wie geht es dir damit im Rückblick?

Sehr gemischt. Prostitution verstößt eigentlich gegen alle Grundsätze einer guten Sexualität und von Gleichberechtigung, es geht häufig um Gewalt und Ausbeutung, sie passiert in einem kriminellen Umfeld. Trotzdem gibt es auch so etwas

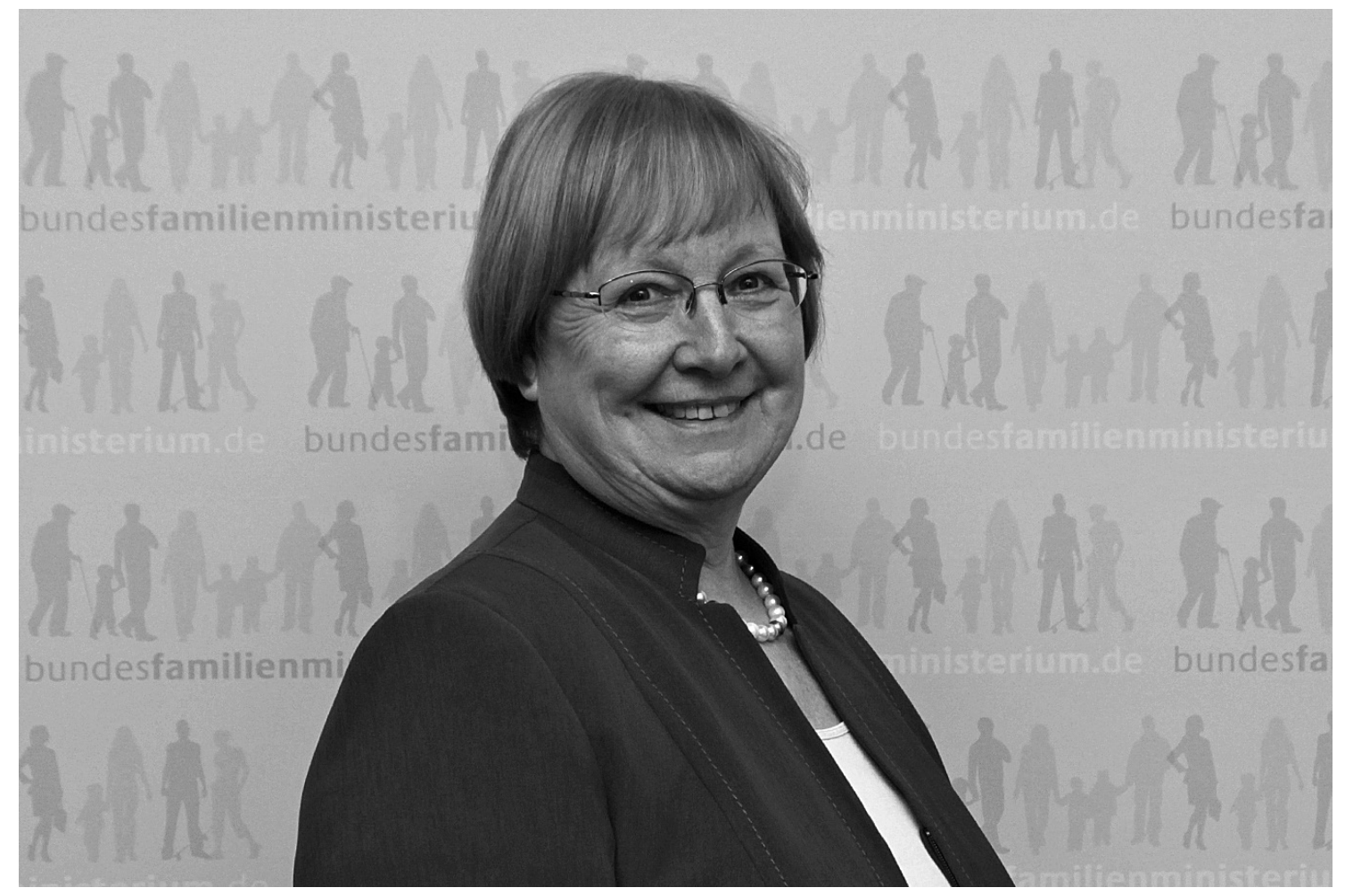

durch die Distanz meines Schreibtisches in gewisser Weise geschützt; ich weiß nicht, ob ich sonst so lange an dem Thema hätte arbeiten können.

Was ich an dem Thema immer mochte ist seine Breite. Es geht um eine Vielzahl von Erscheinungsformen wie sexuelle Belästigung am Arbeitsplatz, häusliche Gewalt, sexueller Missbrauch, Vergewaltigung in der Ehe, Frauenhandel, Genitalver- wie die freiwillige Entscheidung für die Prostitution. Und es gibt Menschen, die aus unterschiedlichen Gründen nur diesen Zugang zu Sexualität haben. Es muss also abgewogen werden zwischen der Achtung des Selbstbestimmungsrechts der Frauen und dem staatlichen Schutz, der dann einsetzen muss, wenn es um Zwang und Ausbeutung geht. Ich meine aber, dass es nicht Aufgabe des Staates ist, Menschen vor (schlechten) Ent- 
scheidungen zu schützen. Andererseits ist er verpflichtet dafür Sorge zu tragen, dass die Rahmenbedingungen die Situation nicht noch verschlimmern. Prostitution muss deshalb aus dem kriminellen Umfeld heraus und sollte „im Hellen“ stattfinden, so dass die Frauen besser geschützt werden können. Was es noch nicht gibt ist, dass Bordellbetreiber einer Genehmigung bedürfen, die mit Auflagen und Weisungen versehen werden kann. Dadurch könnten die Arbeitsbedingungen der Frauen beeinflusst und überwacht werden. Da sind wir damals beim Prostitutionsgesetz auf halbem Weg stehen geblieben - aber mehr war nicht durchzusetzen, und auch in dieser Legislaturperiode haben wir diesen Knoten politisch noch nicht ganz durchschlagen können.

Seit Kurzem bist du Abteilungsleiterin im BMFSFJ. Ich wiederhole hier gerne, was ich auch in der Bund-Länder-Arbeitsgruppe „Gewalt gegen Frauen“ schon gesagt habe: Nicht immer waren die Frauenverbände mit der Arbeit von Familienministerin Schröder zufrieden; zu ihrer Entscheidung, dich zur Abteilungsleiterin zu machen, kann man ihr - und uns aber nur gratulieren (deinen Mitarbeiterinnen und Mitarbeitern, wie ich dich kenne, auch).

Bei aller Freude über deine Ernennung gab es bei mir auch einen zweiten Gedanken: Kurz vor einer Wahl auf den politischen Posten einer Abteilungsleitung zu kommen, heißt vielleicht auch, mit begrenztem Zeithorizont zu arbeiten. Da noch nie ein Frauenminister - ich erinnere an Heiner Geißler - oder eine Frauenministerin nach einer Wahl wieder in das Haus zurück gekehrt ist, könntest du, unabhängig davon, wer die Wahl gewinnt, mit einer neuen Ministerin konfrontiert sein, die durch die Besetzung der Abteilungsleitung einen eigenen Akzent setzen will. Wie gehst du - und wie geht eine Abteilung - mit so diesem „Cliff“ um? Ist da überhaupt eine Überlegung?

Politische Beamte haben immer eine Stellung auf Zeit. Eine neue Abteilungsleitung kommt in der Regel zu Beginn einer neuen Legislatur und steht dann für eine neue Agenda. Eine Ernennung zur Abteilungsleiterin ein gutes Jahr vor der Bundestagswahl ist insofern etwas untypisch. Meine Arbeit konzentriert sich vor allem auf die Umsetzung der bestehenden Agenda. Gleichzeitig erlebe ich es auch als Freiheit, dass ich mich nicht künstlich unter Druck setzen muss, von jetzt auf gleich etwas komplett Neues entwerfen zu müssen.

Was sind aus deiner Sicht die drei großen frauenpolitischen Themen der nächsten Legislatur?

Beim Thema „Gewalt gegen Frauen“ wird die Frage der Absicherung des Hilfesystems auf der Tagesordnung stehen. Wei- tere Themen kreisen um faire Einkommensperspektiven: Entgeltgleichheit, Frauen in Führungspositionen, Wiedereinstieg, aber auch die Anreizsysteme für geringfügige Beschäftigungsverhältnisse werden uns beschäftigen. Ferner wollen gleichstellungspolitische Strategien überlegt sein, wie kann Gleichstellungspolitik zum gesellschaftspolitischen Motor werden? Wie kann es gelingen, den Genderblick in Verwaltung und Politik nachhaltig und durchgängig zu verankern? Auf dem Tisch liegt auch der Erste Gleichstellungsbericht, der in der Analyse wirklich ausgezeichnet ist, aber die Umsetzung seiner Empfehlungen ist nicht so einfach, einige begleiten die Gleichstellungspolitik bereits seit Jahrzehnten und unter wechselnden Regierungen und konnten nicht umgesetzt werden. Es muss also gut bedacht werden: Welche Ziele sind kurz- und mittelfristig überhaupt erreichbar und wie? Das ist eine Herausforderung wie auch die Langlebigkeit von Rollenstereotypen.

Ich weiß, dass du auch einen Plan B hast. In einem Gespräch hast du mal erwähnt, dass du von einer früheren Frauenministerin, Ursula Lehr, die Idee der „Geroprophylaxe“ aufgegriffen hast. Was, wenn ich es richtig verstanden habe, so viel heißt, wie, dass man sich rechtzeitig auf die ,Arbeitslosigkeit' in der Rente vorbereiten soll. Damals sagtest du, dass du u.a. an einem Kommentar mitschreiben wolltest. Und wieder meine Frage: Warum will jemand so was? (Ich habe mich von Kommentaren ferngehalten, weil man die ständig aktualisieren muss.)

Das mit der Geroprophylaxe habe ich mir sehr zu Herzen genommen, das stimmt; aber die Art, wie ich das erreichen möchte, hat sich verändert: Früher habe ich viel geschrieben und gedacht, dass ich das weiter ausbauen würde. Ich habe ja auch tatsächlich an einem Kommentar zum SGB XII mitgeschrieben. Aber ich habe in meiner ehrenamtlichen Arbeit auch gesehen, wie schnell der Kontakt zu Themen verloren geht, wenn man aus den beruflichen Bezügen heraus ist. Deshalb ist Kommentare schreiben heute kein Plan mehr für mein Alter.

Das beruhigt mich! Dann also zum Schluss noch zu Plan C: Du würdest gerne ein Bed \& Breakfast an der englischen Küste aufmachen. Werden djb-Mitglieder dann Rabatt bekommen?

Schau'n wir mal! Dieser Plan besteht weiter und wird - gedanklich - immer weiter gesponnen. Das würde ich wirklich gern machen!

Ich bin sicher, dass die Kommissionen, ständig oder nicht ständig, gern bei dir tagen würden. Danke für das Gespräch. 